\title{
Decay of charged fields in de Sitter spacetime
}

\author{
A. A. Smirnov* \\ Institute of Physics of University of Sao Paulo
}

July 14, 2018

\begin{abstract}
We study the decay of charged scalar and spinor fields around Reissner-Nordström black holes in de Sitter spacetime through calculations of quasinormal frequencies of the fields. The influence of the parameters of the black hole (charge, mass), of the decaying fields (charge, spin), and of the spacetime (cosmological constant) on the decay is analyzed.

The analytic formula for calculation quasinormal frequencies for a large multipole number (eikonal approximation) is derived both for the spinor and scalar fields.
\end{abstract}

\section{Introduction}

It is well known that dynamical evolution of field perturbation on a black hole background can be conventionally divided into three stages [1]. The first stage is an initial wave burst coming directly from the source of perturbation and is dependent on the initial form of the original field perturbation. The characteristic feature of the second stage is the damped oscillations whose frequencies and damping times are defined by the structure of the background spacetime and are independent of the initial perturbation. This stage can be accurately described in terms of the discrete set of quasinormal modes (QNM). And the third stage is an asymptotic tail behavior of the waves at very late time which is caused by backscattering of the gravitational field.

The original interest to the study of QNMs is arising from the possibility to observe quasinormal ringing with the use of gravitational wave detectors as it follows from theoretical predictions (for review see, e.g., [2] and references therein). Recently the interest to the study of QNMs

*e-mail: saa@dfn.if.usp.br 
has been reinforced in connection with their possible relation to the thermodynamic properties of black holes in loop quantum gravity [3, 4].

A new application of the quasinormal mode spectrum has also arisen from superstring theory [5, 6, 7. According to the AdS/CFT correspondence, a large static black hole in asymptotically AdS spacetime corresponds to a thermal state in CFT, and the decay of the test field in the black hole spacetime corresponds to the decay of the perturbed state in CFT, such that the quasinormal frequencies define the thermalization time scale [8]. Therefore, many authors focused their attention on the studies of QNMs for different asymptotically AdS black holes [8, 9, 10, 11, 12, 13.

There exist observational evidences that the universe is described by the general relativity equations with the positive cosmological constant [14, 15, 16]. That observation attracted considerable researches' attention to the study of QNMs in asymptotically de Sitter spacetimes [17, 18, 19, 20, 21, 22, 23, 24, 25].

In [17, 18, the calculation of the quasinormal frequencies for the gravitational perturbations of the Schwarzschild de Sitter (SdS) black hole was carried out. In [22] the lower overtones of QNMs for higher dimensional SdS black holes were calculated. In [24] the total spectrum of QNMs was obtained for the SdS black holes by numerical calculations and the excellent coincidence with the 6th order WKB method was shown. In [23] the low-laying quasinormal frequencies of the SdS black hole for fields of different spin were calculated by the use the 6th order WKB and the Pöshl-Teller potential approximations. In [19] the authors derived an explicit expression for calculation of QNMs for the case of a near extremal SdS black hole. In [20] that expression was generalized to near extremal higher dimensional SdS and Reissner-Nordström de Sitter (RNdS) black holes. In [21] an analytical method was developed to study the quasinormal mode spectrum of SdS black holes for the scalar, electromagnetic, and gravitational fields in the limit of nearly equal black hole and cosmological radii.

The QNMs of the massless uncharged Dirac fields for the RNdS black hole are studied in [25] using the Pöshl-Teller potential approximation. It was found that the magnitude of the imaginary part of the quasinormal frequencies decreases as the cosmological constant or the orbital angular momentum increases, but it increases as the charge or the overtone number increases. We note although that first the Dirac quasinormal modes were evaluated in [26] for Schwarzschild black hole spacetimes.

Perturbations of a charged massless and massive scalar field was by calculation of its QNMs in the Reissner-Nordström (RN), were investigated in [27] and [28], respectively. It was found that the neutral perturbations dominate at the stage of the "final ringdown". In [29, 30] the 
spin 1/2 Dirac particles with the positive, negative, and zero charge $e$ in the presence of the RN black holes with charge $Q$ was investigated. It was demonstrated that in late times the neutral perturbations dominate when $e Q>0$ and the charged perturbations dominate when $e Q<0$.

The decay of fields, which interact electromagnetically with the charge of a black hole in de Sitter spacetime, was not considered till now. The objective of the present paper is to study the decay of charged fields of different spin in RNdS background and analyze how the decay is influenced by a total set of parameters: the charge $Q$ of the black hole, the cosmological constant $\Lambda$, and the charge $e$ of the decaying field.

The paper is organized as follows. In Sect. 2 we consider the spinor field decay, in Sect. 3 we consider the scalar field decay, and in Sect. 4 we summarize the results obtained.

\section{Decay of the spinor field}

In Schwarzschild coordinates, the metric for the Reissner-Nordström de Sitter black hole can be expressed as follows

$$
\begin{gathered}
d s^{2}=-f d t^{2}+f^{-1} d r^{2}+r^{2}\left(d \theta^{2}+\sin ^{2} \theta d \varphi^{2}\right) \\
f=1-\frac{2 M}{r}+\frac{Q^{2}}{r^{2}}-\frac{\Lambda}{3} r^{2}
\end{gathered}
$$

where the parameters $M, Q$ are the mass and the charge of the black hole, respectively, and $\Lambda$ is the cosmological constant. We introduce the tortoise coordinate $r_{*}=\int f^{-1} d r$. Then we write the metric function (2) in the form

$$
f=\frac{\Lambda}{3 r^{2}}\left(r-r_{-}\right)\left(r-r_{+}\right)\left(r_{c}-r\right)\left(r-r_{b}\right)
$$

where $r_{c}$ is the cosmological horizon, $r_{-}$is the inner event horizon, and $r_{+}$is the outer event horizon $\left(r_{b}\right.$ can be found through $\left.r_{-}, r_{+}, r_{c}\right)$. One can find that the tortoise coordinate $r_{*}$ can be expressed as follows

$$
\begin{aligned}
& r_{*}=\frac{1}{2}\left[\frac{1}{\kappa_{-}} \ln \left(r-r_{-}\right)+\frac{1}{\kappa_{+}} \ln \left(r-r_{+}\right)\right. \\
& \left.-\frac{1}{\kappa_{c}} \ln \left(r_{c}-r\right)+\frac{1}{\kappa_{b}} \ln \left(r-r_{b}\right)\right] \\
& \kappa_{a}=\left.\frac{1}{2} \frac{d f}{d r}\right|_{r=r_{a}}, a=(-,+, c, b) .
\end{aligned}
$$

From (3) one can see that $r_{*} \rightarrow \infty$ as $r \rightarrow r_{c}$ and $r_{*} \rightarrow-\infty$ as $r \rightarrow r_{+}$. 
The wave equation of the massless Dirac field can be written as

$$
\left[\gamma^{a} e_{a}^{\mu}\left(\partial_{\mu}+\Gamma_{\mu}+e A_{\mu}\right)\right] \Psi=0
$$

where $\gamma^{a}$ are the Dirac matrices, $e_{a}^{\mu}$ is the inverse of the tetrad $e_{\mu}^{a}, \Gamma_{\mu}=\frac{1}{8}\left[\gamma^{a}, \gamma^{b}\right] e_{a}^{\nu} e_{b \nu ; \mu}$ is the spin connection. We take the tetrad $e_{\mu}^{a}$ as [31]

$$
e_{\mu}^{a}=\operatorname{diag}\left(f^{1 / 2}, f^{-1 / 2}, r, r \sin \theta\right) .
$$

We define $\Phi=f^{-1 / 4} \Psi$, then using the ansatz

$$
\Phi=\left(\begin{array}{c}
\frac{i G^{( \pm)}(r)}{r} \phi_{j m}^{ \pm}(\theta, \varphi) \\
\frac{F^{( \pm)}(r)}{r} \phi_{j m}^{\mp}(\theta, \varphi)
\end{array}\right) e^{-i \omega t}
$$

where

$$
\begin{gathered}
\phi_{j m}^{+}=\left(\begin{array}{c}
\sqrt{\frac{j+m}{2 j}} Y_{l}^{m-1 / 2} \\
\sqrt{\frac{j-m}{2 j}} Y_{l}^{m+1 / 2}
\end{array}\right) \text { for } j=l+\frac{1}{2} \\
\phi_{j m}^{-}=\left(\begin{array}{c}
\sqrt{\frac{j+1-m}{2 j+2}} Y_{l}^{m-1 / 2} \\
-\sqrt{\frac{j+1+m}{2 j+2}} Y_{l}^{m+1 / 2}
\end{array}\right) \text { for } j=l-\frac{1}{2}
\end{gathered}
$$

we find for both signs of $F$ and $G$,

$$
\begin{aligned}
& \frac{d^{2} F}{d r_{*}^{2}}+\left(\omega^{2}-V_{1}\right) F=0 \\
& \frac{d^{2} G}{d r_{*}^{2}}+\left(\omega^{2}-V_{2}\right) G=0
\end{aligned}
$$

where

$$
\begin{aligned}
& V_{1,2}= \pm \frac{d W}{d r_{*}}+W^{2}, W=\frac{|k| \sqrt{f}}{r}-\frac{e Q}{r}, \\
& k=j+\frac{1}{2}, j=l+\frac{1}{2} \text { for } V_{1}, \\
& k=-\left(j+\frac{1}{2}\right), j=l-\frac{1}{2} \text { for } V_{2} .
\end{aligned}
$$

The quasinormal modes are defined as solutions of (77), (8) satisfying the boundary conditions

$$
F\left(r_{*}\right) \sim A_{ \pm} e^{ \pm i \omega r_{*}}, G\left(r_{*}\right) \sim B_{ \pm} e^{ \pm i \omega r_{*}}, r_{*} \rightarrow \pm \infty
$$

supposing Rew $>0$, that corresponds to purely in-going waves at the event horizon and purely out-going waves the cosmological horizon.

We notice that the potentials $V_{1}, V_{2}$ give the same quasinormal frequencies [25], [30], therefore in what follows we focus the attention on $V_{1}$. The effective potential $V_{1}$ (91) is a smooth 


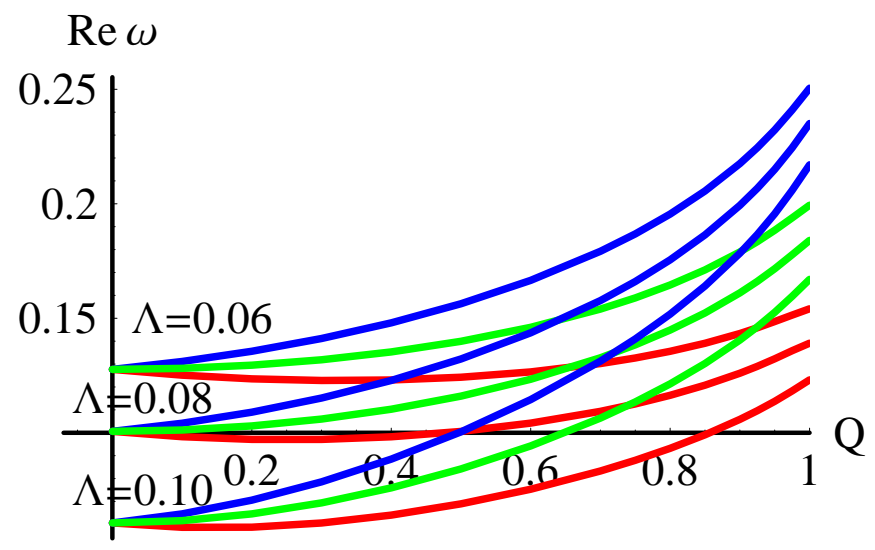

Figure 1: QN frequencies of the spinor field; red: $e=0.1$, blue: $e=-0.1$, green: $e=0$.

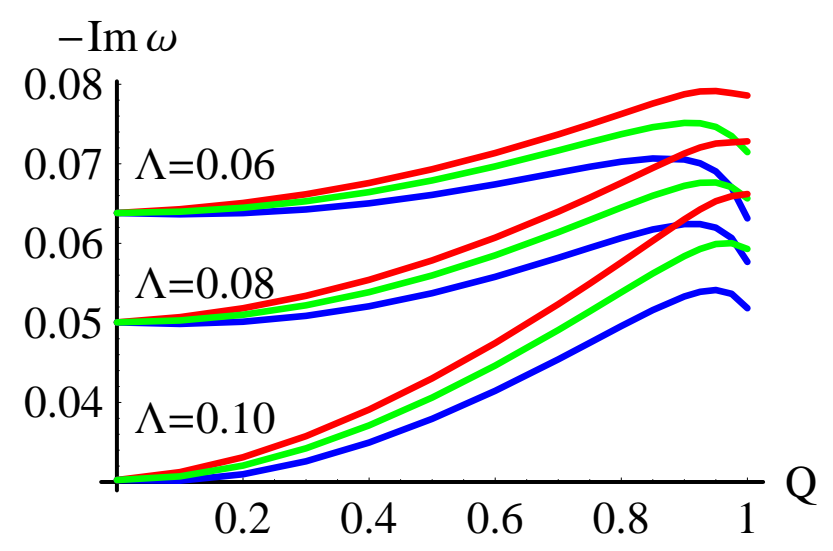

Figure 2: Imaginary part of $\omega$ of the spinor field; red: $e=0.1$, blue: $e=-0.1$, green: $e=0$.

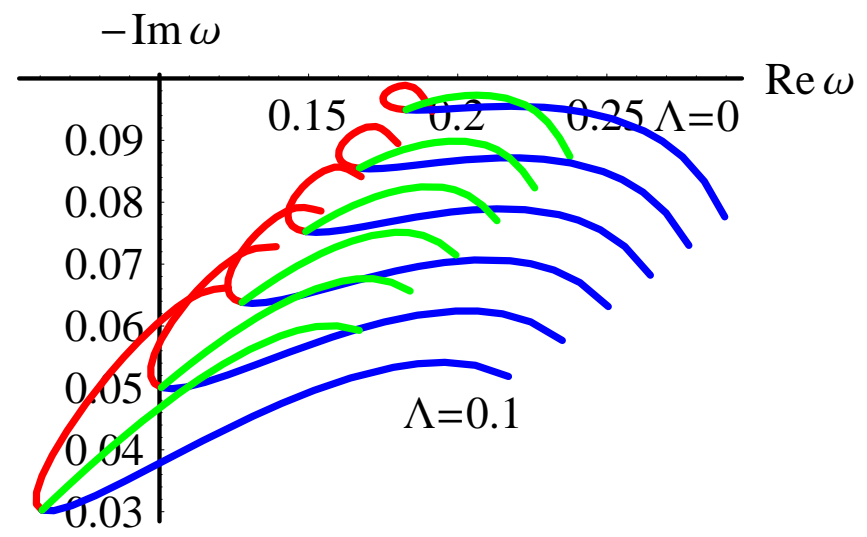

Figure 3: QNM of the spinor field parametrized by $Q$ for $\Lambda=0,0.2,0.4,0.6,0.8,0.1$; red: $e=0.1$, blue: $e=-0.1$, green: $e=0$.

function of $r_{*}$. It tends to constant values at the cosmological horizon and at the outer event horizon and has a maximum near the outer event horizon that allows to use the WKB method to calculate quasinormal modes. To compute QNMs from Eqs. (7), (9) we apply the WKB 


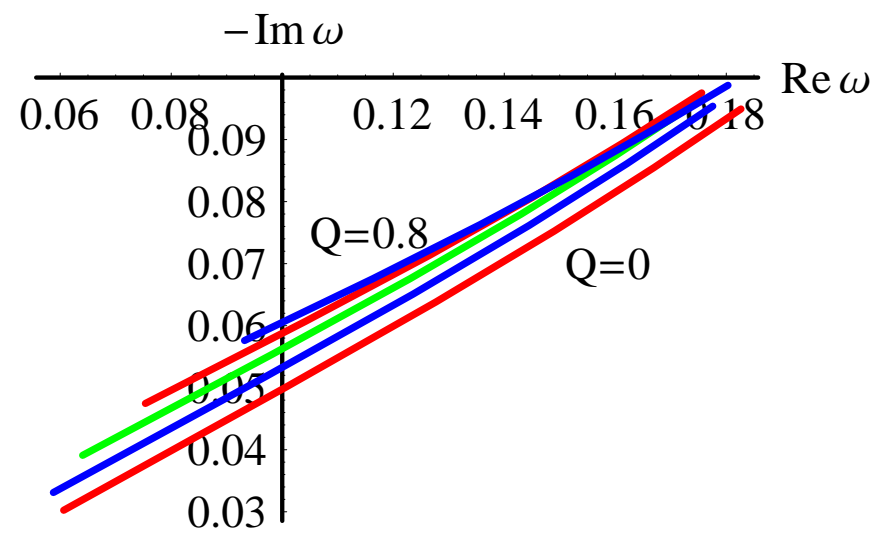

Figure 4: QNM of the spinor field parametrized by $\Lambda$ for $e=0.1, Q=0,0.2,0.4,0.6,0.8$.

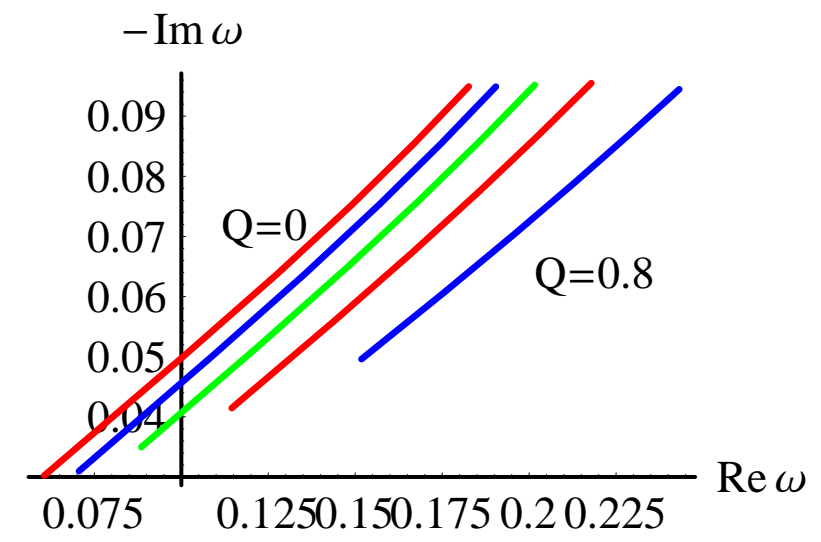

Figure 5: QNM of the spinor field parametrized by $\Lambda$ for $e=-0.1, Q=0,0.2,0.4,0.6,0.8$.

method of the sixth order [32].

We present graphically the results for the fixed overtone number $n=0$ and $k=1$ at Figs. 1-5 for the charge of the spinor field $e=0, \pm 0.1$. It is shown the dependence of QN frequencies (Fig. 1) and their damping rates (Fig. 2) on the charge of the black hole $Q$ for different values of the cosmological constant $\Lambda$. On Figs. 1, 2 the dependences are shown for three values of the charge of the spinor field $e=0, \pm 0.1$, which on the figures looks like a triplet of curves beginning at the same point at $Q=0$. The curves of different densities on the graphs correspond to different $e$. On Fig. 1 the upper curve in the triplets corresponds to $e=-0.1$, the lower curve in the triplets corresponds to $e=0.1$, the middle curve corresponds to $e=0$. On Fig. 2 the lower curve in the triplets corresponds to $e=-0.1$, the upper curve in the triplets corresponds to $e=0.1$, the middle curve corresponds to $e=0$. The $\mathrm{QN}$ frequencies are monotonically growing with $Q$ for $e=0,-0.1$, although for $e=0.1$ the behavior of the curves is qualitatively different. The curves for $e=0.1$ have a minimum at some point $Q_{0}$. 
The value of $Q_{0}$ is shifting to less values as $\Lambda$ grows. Besides, Fig. 1 demonstrates that the QN frequencies become lower as $\Lambda$ increasing. The position of a curve in the triplet says that the QN frequencies is lower for the field charge $e>0$ and higher for $e<0(Q>0)$. The curves displaying QNM damping rate are similar for all $\Lambda$ and $e$. They have a maximum at $Q_{\max } \sim 0.9$. Until reaching $Q_{\max }$ the curves are slowly growing and after reaching $Q_{\max }$ they are sharply falling down. The greater $\Lambda$ is the greater the rise of the curves is until reaching $Q_{\max }$. The value of $Q_{\max }$ is shifting to greater values as $\Lambda$ increasing. The position of a curve in the triplet says that the decay of the field is slower for the field charge $e<0$ and faster for $e>0(Q>0)$. The decay of the spinor fields becomes slower as $\Lambda$ grows. Since the charges $e, Q$ appear in $V_{1}$ only as $e^{2}, Q^{2}$ or $e Q$ one can also conclude that for $Q<0$ the conclusions become upside-down. These observations generalize results the work [30] obtained for asymptotically flat spacetimes. The peculiarities of the dependence of $\operatorname{Im} \omega$, $\operatorname{Re} \omega$ on $Q$ are more obvious when depicted on diagrams of the dependence of $\operatorname{Im} \omega$ on Rew and parametrized by the black hole charge $Q$. These curious pictures are presented on Fig. 3.

The dependence of $\operatorname{Im} \omega$ on Re $\omega$ is parametrized by the cosmological constant $\Lambda$, which is changing along the curves, is depicted on Figs. 4, 5 for the fixed black hole charge $Q$. The curves are approximately straight lines. This observation generalizes conclusions the research 25. obtained for the uncharged spinor field. Both the QN frequencies and their damping rates are decreasing with $\Lambda$, although the manner of the decrease for Re $\omega$ and $\operatorname{Im} \omega$ is different.

Making use of the first order WKB method we also found the asymptotic formula of large $k$ for calculation quasinormal modes $\omega$,

$$
\omega=C_{0} k-i(n+1 / 2) C_{i}^{s p}+C_{r}^{s p}+O(1 / k)
$$

where

$$
\begin{aligned}
& C_{0}=\left(\frac{Q^{2}}{r_{0}^{4}}-\frac{2 M}{r_{0}^{3}}+\frac{1}{r_{0}^{2}}-\frac{\Lambda}{3}\right)^{1 / 2} \\
& C_{i}^{s p}=\frac{1}{\sqrt{3} r_{0}^{3}}\left[-42 Q^{4}+3 Q^{2} r_{0}\left(42 M-15 r_{0}+2 \Lambda r_{0}^{3}\right)+\right. \\
& \left.r_{0}^{2}\left(90 M^{2}-60 M r_{0}+9 r_{0}^{2}+6 M \Lambda r_{0}^{3}-\Lambda r_{0}^{4}\right)\right]^{1 / 2} \\
& C_{r}^{s p}=-\frac{1}{2 r_{0}^{2}}\left[4 r_{1}^{s p} r_{0} C_{0}^{1 / 2}+\left(r_{0}-3 M\right)+\right. \\
& \left.\frac{2}{3 r_{0}^{2}} C_{0}^{-1 / 2}\left(Q^{2}+e Q r_{0}^{2}+r_{1}^{s p}\left(3 M-3 r_{0}+2 r_{0}^{3} \Lambda\right)\right)\right]
\end{aligned}
$$




$$
\begin{aligned}
& r_{0}=\frac{3}{2} M\left(1+\sqrt{1-\frac{8 Q^{2}}{9 M^{2}}}\right), \\
& r_{1}^{s p}=-\frac{1}{2} r_{0}^{2} C_{0}^{1 / 2} \\
& \left(-30 Q^{4}+90 M Q^{2} r_{0}-63 M^{2} r_{0}^{2}-33 Q^{2} r_{0}^{2}\right. \\
& -18 e Q^{3} r_{0}^{2}+42 M r_{0}^{3}+30 e M Q r_{0}^{3}-6 r_{0}^{4} \\
& \left.+12 e Q r_{0}^{4}+6 Q^{2} r_{0}^{4} \Lambda-6 M r_{0}^{5} \Lambda+r_{0}^{6} \Lambda+2 e Q r_{0}^{6} \Lambda\right) \\
& \left(-42 Q^{4}+126 M Q^{2} r_{0}-90 M^{2} r_{0}^{2}-45 Q^{2} r_{0}^{2}\right. \\
& \left.+60 M r_{0}^{3}-9 r_{0}^{4}+6 Q^{2} r_{0}^{4} \Lambda-6 M r_{0}^{5} \Lambda+r_{0}^{6} \Lambda\right)^{-1} .
\end{aligned}
$$

By setting $Q=0$ one can make sure that the formula (11) reproduces the corresponding formula of the paper 23] obtained for the SdS black hole. We note that Im $\omega$ does not depend on the charge of the spinor field $e$ in the limit of large $k$. Therefore the damping rate is equal for the arbitrary spinor field charge for large enough multipole moment. The dependence of Re $\omega$ on $k$ is linear for large $k$. The slope of the line on the plane $(\operatorname{Re} \omega, k)$ is defined by the coefficient $C_{0}$. These conclusions are confirmed by the results of [30] where the particular case $\Lambda=0$ was considered.

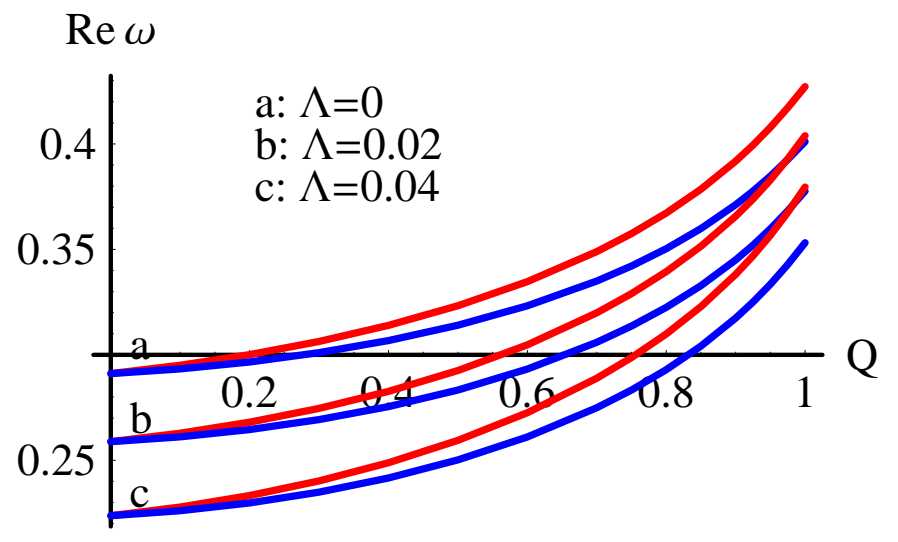

Figure 6: QN frequencies of the scalar field; red: $e=0.1$, blue: $e=0.05$.

\section{Decay of the scalar field}

The wave equation of the complex scalar field has the form 33 ,

$$
\phi_{; a b}-i e A_{a} g^{a b}\left(2 \phi_{; b}-i e A_{b} \phi\right)-i e A_{a ; b} g^{a b}=0
$$

Here we choose the electromagnetic potential as $A_{t}=-Q / r$. Then, decomposing the wave function $\phi$ into spherical harmonics $\phi=\sum_{l, m} u_{m}^{l}(t, r) Y_{m}^{l}(\theta, \varphi) / r$, the wave equation for 


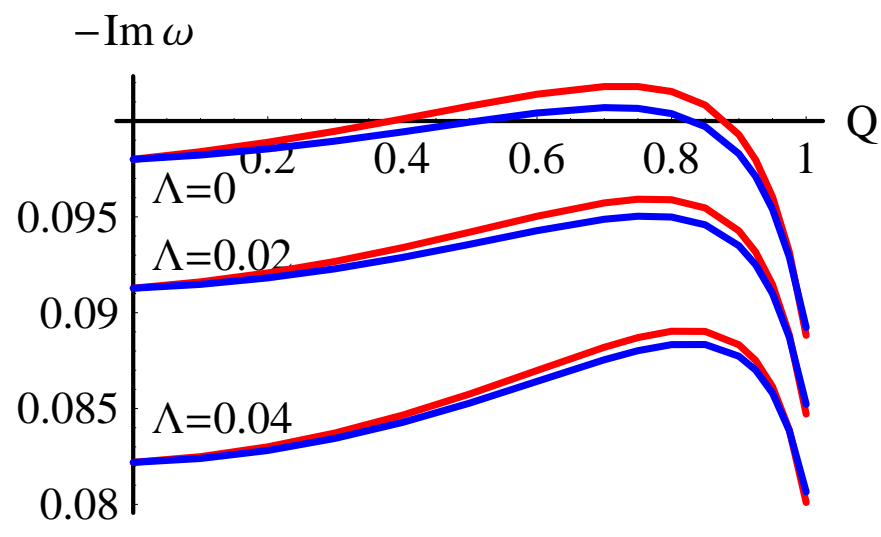

Figure 7: Imaginary part of $\omega$ of the scalar field; red: $e=0.1$, blue: $e=0.05$.

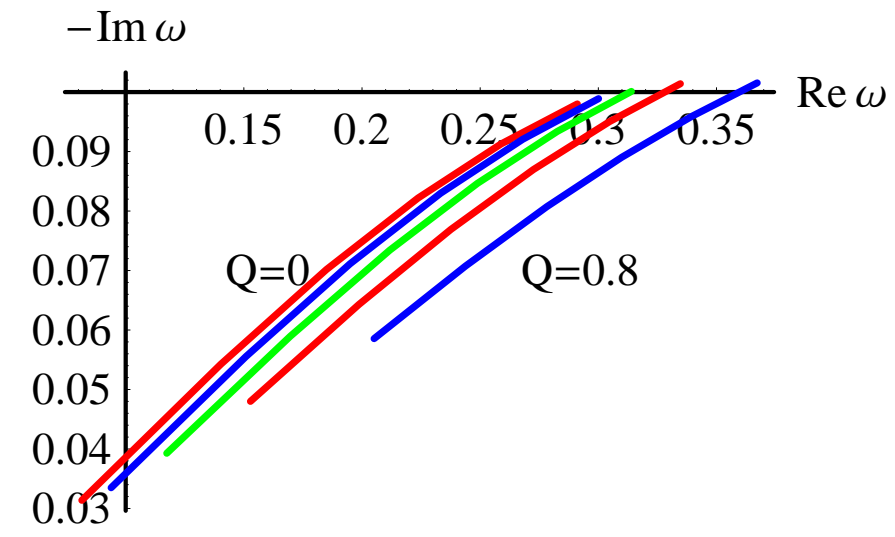

Figure 8: QNM of the scalar field parametrized by $\Lambda$ for $Q=0,0.2,0.4,0.6,0.8 ; e=0.1$.

each multipole moment takes the form

$$
\begin{gathered}
\partial_{t}^{2} u+2 i e \frac{Q}{r} \partial_{t} u-\partial_{r_{*}}^{2} u+V u=0 \\
V=f\left(\frac{l(l+1)}{r}+\frac{2 M}{r^{3}}-\frac{2 Q^{2}}{r^{4}}-\frac{2 \Lambda}{3}\right)-\frac{e^{2} Q^{2}}{r^{2}}
\end{gathered}
$$

where $r_{*}$ is the tortoise coordinate and $u(t, r)=e^{-i \omega t} u(r)$. Therefore we come to the equation for $u(r)$,

$$
\begin{aligned}
& \partial_{r_{*}}^{2} u+\left[\omega^{2}-V_{e f f}\right] u=0, \\
& V_{e f f}=V+\frac{2 e Q \omega}{r} .
\end{aligned}
$$

Supposing Rew $>0$ solutions of (17) have to satisfy the boundary conditions

$$
u\left(r_{*}\right) \sim C_{ \pm} e^{ \pm i \omega r_{*}}, r_{*} \rightarrow \pm \infty .
$$


To calculate quasinormal modes we apply the WKB method of the third order [34] in the scalar field case. The motivation about the use of WKB method is the same as that in Sect. 2. The use of the calculation method of higher order 32] is overly cumbersome as in the case of scalar field $V_{\text {eff }}$ includes $\omega$ so that it is necessary to solve simultaneously the equation for the search of an extremum and the equations of the WKB method itself.

We present graphically the results for the fixed overtone number $n=0$ and multipole number $l=1$ on Figs. 6-8. It is shown the dependence of QN frequencies (Fig. 6) and their damping rates (Fig. 7) on the charge of the black hole $Q$ for different values of the cosmological constant $\Lambda$. On Figs. 6, 7 the dependences are shown for two values of the charge of the scalar field $e=0.1,0.05$, which on the figures looks like a couple of curves beginning at the same point at $Q=0$. The upper curve in the couples corresponds to $e=0.1$, the lower curve lower one of the couples corresponds to $e=0.05$.

The QN frequencies monotonically grow with $Q$ and become lower as $\Lambda$ increasing. The QN frequencies are higher for the fields with greater charge $e$. The curves displaying the QNM damping rates have a pronounced maximum which is located at $Q_{\max } \simeq 0.8$ and a location of the maximum is shifting from $Q \simeq 0.7$.to $Q \simeq 0.9$ as $\Lambda$ increasing. Until reaching $Q_{\max }$ the curves are slowly growing and after reaching $Q_{\max }$ they are sharply flowing down. Fig. 7 shows that the field decays faster if it has greater charge. And similar to the spinor case the decay of the scalar fields becomes slower as $\Lambda$ grows. Both the QN frequencies and the damping rates are decreasing with $\Lambda$, although the manner of the decrease is different.

Fig. 8 displays QNMs for different fixed $Q$. The curves on Fig. 8 are parametrized by the cosmological constant which is changing along the curves. We note that $\operatorname{Im} \omega$ is related to Re $\omega$ not linearly in contrast to the spinor field case (Figs. 4, 5).

Making use of the first order WKB method it is also possible to find the asymptotic formula of large $l$ for calculation quasinormal modes $\omega$ for the scalar field,

$$
\omega=C_{0}(l+1 / 2)-i(n+1 / 2) C_{i}^{s c}+C_{r}^{s c}+O(1 / l)
$$

where

$$
\begin{aligned}
& C_{i}^{s c}=\frac{1}{3} r_{0}^{-3}\left\{-126 Q^{4} r_{0}^{2}+Q^{2} r_{0}^{2}\left[378 M r_{0}+r_{0}^{2}\left(-135+18 \Lambda r_{0}^{2}\right)\right]+\right. \\
& \left.r_{0}^{3}\left[-270 M^{2} r_{0}-6 M r_{0}^{2}\left(-30+3 \Lambda r_{0}^{2}\right)+r_{0}^{3}\left(-27+3 \Lambda r_{0}^{2}\right)\right]\right\}^{1 / 2}, \\
& C_{r}^{s c}=\frac{r_{1}^{s c}}{r_{0}^{3}}\left(-1-\frac{2 Q^{2}}{r_{0}^{2}}+\frac{3 M}{r_{0}}\right) C_{0}^{-1},
\end{aligned}
$$




$$
\begin{aligned}
& r_{1}^{s c}=-r_{0}\left(-6 Q^{4}+21 M Q^{2} r_{0}-18 M^{2} r_{0}^{2}-9 Q^{2} r_{0}^{2}+\right. \\
& \left.15 M r_{0}^{3}-3 r_{0}^{4}+2 Q^{2} \Lambda r_{0}^{4}-3 M \Lambda r_{0}^{5}+\Lambda r_{0}^{6}\right) \times \\
& \left(42 Q^{4}-12 M Q^{2} r_{0}+90 M^{2} r_{0}^{2}+45 Q^{2} r_{0}^{2}\right. \\
& \left.-60 M r_{0}^{3}+9 r_{0}^{4}-6 Q^{2} \Lambda r_{0}^{4}+6 M \Lambda r_{0}^{5}-\Lambda r_{0}^{6}\right) .
\end{aligned}
$$

In expressions (19)-(21) $C_{0}, r_{0}$ are the same as those in formulas (12), (131) of Sect. 2. By setting $Q=0$ one can make sure that the formula (11) reproduces the corresponding formula of the paper [23] obtained for the SdS black hole for the scalar field. We note that the asymptotic formula for the RN black hole in asyptotically flat spacetime for the charged scalar field was not found till now. The formlula for such a particular case can be obtained by setting $\Lambda=0$ in (19)-(21).

\section{Conclusions}

We considered the decay of the charged spinor and scalar field near the Reissner-Nordström black hole in de Sitter spacetime. We calculated the quasinormal frequencies of the fields and their damping rates for the lower overtone number. The analysis of the results allows to formulate the following conclusions. a) In the spinor case for a fixed cosmological constant $\Lambda$ the quasinormal frequencies of the charged field are lower and the damping rates are higher than those of the neutral field if $e Q>0$ and vice versa if $e Q<0$; b) In the scalar case for a fixed cosmological constant $\Lambda$ the fields possessing greater charge have higher quasinormal frequencies and decay faster; c) The quasinormal frequencies of both the spinor and scalar fields are lower in the spacetimes with greater cosmological constant $\Lambda$. The decay of both the spinor and scalar fields is slower in the spacetimes with greater cosmological constant $\Lambda$; d) In the case of the charged spinor field the dependence of $\operatorname{Im} \omega$ on Rew is approximately linear on the diagram parametrized by $\Lambda$; e) In the case of the charged scalar field the dependence of $\operatorname{Im} \omega$ on Re $\omega$ is not linear on the diagram parametrized by $\Lambda$;) The dependence of $\operatorname{Im} \omega$ and Rew of the charged spinor field on the black hole charge $Q$ is different for $e>0$ and $e<0$ for all values of $\Lambda$.

We derived the analytic formula for calculation of $\omega$ for large values of the multipole number both for the spinor and scalar fields. The formula for the spinor field shows that for asymptotically large multipole number the damping rate does not depend on the charge $e$ of the spinor field. 


\section{References}

[1] V. P. Frolov, I.. D. Novikov, Black hole physics: basic concepts and new developments (Kluwer Academic publishers, 1998).

[2] K. Kokkotas and B. Schmidt, Liv. Rev. Rel. 2, 2 (1999); H. P. Nollert, Class. Quant. Grav.16, R159 (1999).

[3] S. Hod, Phys. Rev. Lett. 81, 4293 (1998).

[4] O. Dreyer, Quasinormal Modes, Phys. Rev. Lett. 90, 081301 (2003).

[5] J. Maldacena, Adv. Theor. Math. Phys. 2, 231 (1998).

[6] E. Witten, Adv. Theor. Math. Phys. 2, 253 (1998).

[7] S. Kalyana Rama and B. Sathiapalan, Mod. Phys. Lett. A 14, 2635 (1999).

[8] G. T. Horowitz and V. E. Hubeny, Phys. Rev. D 62, 024027 (2000).

[9] V. Cardoso and J. P. S. Lemos, Phys. Rev. D 63, 124015 (2001).

[10] D. Birmingham, I. Sachs, and S. N. Solodukhin, Phys. Rev. Lett. 88, 151301 (2002).

[11] R. A. Konoplya, Phys. Rev. D 66, 044009 (2002); hep-th/0205142.

[12] V. Cardoso, R. Konoplya, and J. P. S. Lemos, Phys. Rev. D 68, 044024 (2003); gr-qc/0305037.

[13] R.A. Konoplya, Phys. Rev. D 70, 047503 (2004); hep-th/0406100.

[14] S. Perlmutter et al., Astrophys. J. 483, 565 (1997).

[15] R. R. Caldwell, R. Dave, and P. J. Steindardt, Phys. Rev. Lett. 80, 1582 (1998).

[16] P. M. Garnavich et al., Astrophys. J. 509, 74 (1998).

[17] H. Otsuki and T. Futamase, Prog. Theor. Phys. 85, 771 (1991).

[18] I. G. Moss and J. P. Norman, Class. Quant. Grav. 19, 2323 (2002).

[19] V. Cardoso and J. P. S. Lemos, Phys. Rev. D 67, 084020 (2003).

[20] C. Molina, Phys. Rev. D 68, 064007 (2003). 
[21] A. Maassen van den Brink, Phys. Rev. D 68, 047501 (2003).

[22] R.A. Konoplya, Phys. Rev. D 68, 124017 (2003); hep-th/0309030.

[23] A. Zhidenko, Class. Quant. Grav. 21, 273 (2004); gr-qc/0307012.

[24] R. A. Konoplya, A. Zhidenko, JHEP 0406, 037 (2004); hep-th/0402080.

[25] J. Jing, Phys. Rev. D 69, 084009 (2004); gr-qc/0312079.

[26] H. T. Cho, Phys. Rev. D 68, 024003 (2003).

[27] R. A. Konoplya, Phys. Rev. D 66, 084007 (2002); gr-qc/0207028

[28] R.A. Konoplya, Phys. Lett. B 550, 117 (2002); gr-qc/0210105.

[29] H. Zhang and Wei Zhou, Class.Quant.Grav. 21, 917 (2004); gr-qc/0312029.

[30] Wei Zhou and Jian-Yang Zhu, Int. J. Mod. Phys. D 13, 1105 (2004); gr-qc/0309071.

[31] D. R. Brill and J. A. Wheeler, Rev. Mod. Phys. 29, 465 (1957).

[32] R. A. Konoplya, Phys. Rev. D 68, 024018 (2003); gr-qc/0303052.

[33] S. W. Hawking, G. F. R. Ellis, The Large Scale structure of Space Time (Cambridge University Press, Cambridge, England,1973).

[34] S. Iyer and C. M. Will, Phys. Rev. D 35, 3621 (1987). 